\title{
Implementación de Central Telefónica Asterisk sobre Raspberry incluyendo Tarifación y Seguridad para el Usuario
}

Implementation of Asterisk (PBX) on Raspberry including Pricing and User

Security

Implantação de Central Telefonica Asterisk on framboesa incluindo o carregamento e segurança do usuário

DOI: $\underline{\text { http://dx.doi.org/10.23913/reci.v6i11.61 }}$

Kathy Valeria Salgado Mantilla

Universidad de las Fuerzas Armadas - ESPE, Ecuador

kvsalgado@espe.edu.ec

Freddy Roberto Acosta Buenaño

Universidad de las Fuerzas Armadas - ESPE, Ecuador

fracosta@espe.edu.ec

Christian Israel Fiallos Silva

Universidad de las Fuerzas Armadas - ESPE, Ecuador

cifiallos@espe.edu.ec

Andrés Ricardo González Hernández

Universidad de las Fuerzas Armadas - ESPE, Ecuador agonzalez@espe.edu.ec

Raúl Vinicio Haro Báez

Universidad de las Fuerzas Armadas - ESPE, Ecuador rharo@espe.edu.ec 


\section{Resumen}

En este artículo se encuentra la descripción de un sistema completo de comunicación donde se saca provecho a todas las particularidades que una central telefónica pueda ofrecer, dirigida especialmente para medianas o pequeñas empresas que busquen el mejor sistema de comunicación por VoIP a un menor costo. Se destacan aspectos como la utilización de software gratuito de telefonía disponible, como Asterisk, para la implementación de la central telefónica y un sistema de tarifación con licencia libre llamado Servitux, el cual se utilizó para el control y cobro de cada llamada externa, también se manejó hardware de bajo costo, como es la tarjeta Raspberry Pi, donde se buscó aprovechar las ventajas del sistema operativo dando más seguridad por medio de programación en lenguaje php y html, brindando la posibilidad del cambio de clave para cada una de las diferentes extensiones en la central. Finalmente se realizan pruebas de estrés utilizando el software de licencia libre SIPp, definiendo la capacidad del sistema. También por medio de un análisis MOS queda demostrado que se puede satisfacer las necesidades del cliente en cuanto a la calidad y confiabilidad en el servicio de voz QoE.

Palabras clave: software, telefonía, Servitux, Asterisk, Raspberry, comunicación.

\section{Abstract}

This article is the description of a complete communication system where benefits to all the characteristics that a Business Telephone System can offer, especially for medium-sized or small companies who are seeking the best system of VoIP communication at lower cost. We highlights aspects as the use of free software available telephony, as Asterisk, for the implementation of the telephone exchange and a system of charging licensed free called Servitux, which was used for the control and payment of each external call, we also managed low-cost hardware, as the card is Raspberry Pi, where we sought to take advantage of operating system giving more security through programming in php and html language, providing the possibility of the change of key to each of the different extensions in the Private Branch Exchange (PBX). Finally there are stress tests using the SIPp free license software, defining the capacity of the system. Also using a MOS analysis are shown which 
can satisfy the needs of the customer in terms of quality and reliability in voice Quality of Experience (QoE).

Key words: software, telephony, Servitux, Asterisk, Raspberry, communication.

\section{Resumo}

Este artigo é a descrição de um sistema de comunicação completo onde ele tira proveito de todas as características que um call center pode oferecer, destinadas especialmente para as empresas médias ou pequenas que procuram o melhor sistema VoIP de comunicação a um custo menor. aspectos como o uso de software de telefonia livre disponíveis, tais como Asterisk, para a implementação da troca de telefone e sistema de carregamento com licença livre chamado Servitux, que foi usado para o controlo e recolha de cada estande chamada externa, também manipulados hardware de baixo custo, como a placa Raspberry Pi, onde procurou para tirar proveito do sistema operacional dando mais segurança através de programação PHP e HTML, oferecendo a possibilidade de mudar chave para cada um dos diferentes extensões central. Finalmente salientar testes são realizados usando o SIPp licença de software livre, definindo a capacidade do sistema. Também através da análise MOS demonstramos que pode atender às necessidades dos clientes em termos de qualidade e confiabilidade no serviço de voz QoE.

Palavras-chave: software, telefonia, Servitux, Asterisk, framboesa, comunicação.

Fecha Recepción: 2017 Fecha Aceptación: 2017 


\section{Introducción}

La red telefónica pública conmutada PSTN (del inglés Public Switched Telephone Network) es una arquitectura bastante tradicional y compleja utilizada en sistemas de comunicación. En la actualidad es notable el crecimiento del uso de internet, el cual ha brindado la posibilidad de integrar la telefonía e implementarla a nivel de software, también llamado VoIP (Voice Over Internet Protocol), reduciendo costos y complejidad, siendo uno de los servicios más importantes prestados a través de redes de información, incluso es adaptable a sistemas tradicionales de comunicación como la red telefónica pública conmutada PSTN (Sinaeepourfard y Mohamed, 2011). Por lo tanto, las diferentes propuestas de Voz sobre IP no sólo se encuentran enfocadas a grandes empresas, sino también medianas, pequeñas y diferentes entornos domésticos.

En la actualidad existen varias aplicaciones y programas que se utilizan para implementar una central telefónica. En el mercado se encuentran aquellos que conllevan un costo tanto de adquisición como de uso, pero también existen aquellos que se encuentran disponibles en la red sin costo alguno.

Dentro de los programas disponibles y sin costo para la implementación de una central telefónica esta Elastix, configurable mediante una interfaz gráfica, y Asterisk, basado en código abierto de Linux. Estos programas son bastante manejables, estables, rentables (Qadeer e Imran, 2008) y se los pueden emplear sobre diferentes tipos de hardware (Pelaéz y Tipantuña, 2014), permitiendo obtener una variedad de beneficios incorporando varias alternativas de comunicación unificando servicios de telefonía como, correo electrónico, correo de voz, comunicación interna, todo en una misma plataforma (Dong, 2011; Masudur, M., y Sarwar, N., 2014).

Para determinar la calidad en la comunicación que se desarrolla dentro de una central telefónica que utilice Asterisk como software, se realizan pruebas de análisis o configuraciones sobre diferentes características de la central, generalmente enfocándose en la comunicación interna entre extensiones (Li, Li, Wang y Nan, 2011). 
Existe una variedad de sistemas embebidos, entre ellos la tarjeta Alix o Raspberry Pi, los cuales pueden ser utilizados como hardware de bajo costo (Villacis, Acosta, y Lara, 2013), para desarrollar y aprovechar al máximo todos los beneficios que Asterisk brinda como central telefónica (Estrada, Peláez y Tipantuña, 2015; Murkute \& Deshmukh, 2015). Es posible incluir un Gateway de voz en el hardware del sistema, que permitirá que exista comunicación externa permitiendo la entrada y salida de llamadas, desde y hacia la red telefónica PSTN (del inglés Public Switched Telephone Network) (Gupta, Agrawal y Qadeer, 2013).

El objetivo del presente artículo es mostrar una central telefónica completa que puede ser implementada específicamente en pequeñas y medianas empresas, dando especial importancia al software y hardware de bajo costo usando Asterisk sobre la tarjeta Raspberry $\mathrm{Pi}$, obteniendo así comunicación interna entre extensiones donde experimentalmente se incluirá por única vez un Gateway de voz para que exista comunicación externa, y se tomara en cuenta la seguridad en el uso del sistema mediante el cambio de claves para el acceso a cada extensión cada vez que se crea necesario, también se mantendrá un control de facturación y registro para las llamadas externas realizadas por el personal y clientes de la empresa.

\section{Diseño e Implementación del Sistema}

\section{Caso de estudio: Hotel}

Para la implementación del sistema se utilizó el siguiente hardware:

- Ordenador o placa reducida Raspberry Pi

- Gateway de voz Ht503

- Router

La implementación consiste en la configuración de una central telefónica con el software libre Asterisk (Asterisk.org, 2017) que usa una misma red dentro del establecimiento, incluyendo servicios de telefonía, correo de voz y además permite llamadas tanto internas entre extensiones, como externas hacia la red pública convencional. 
Figura 1. Diagrama de bloque del sistema implementado.

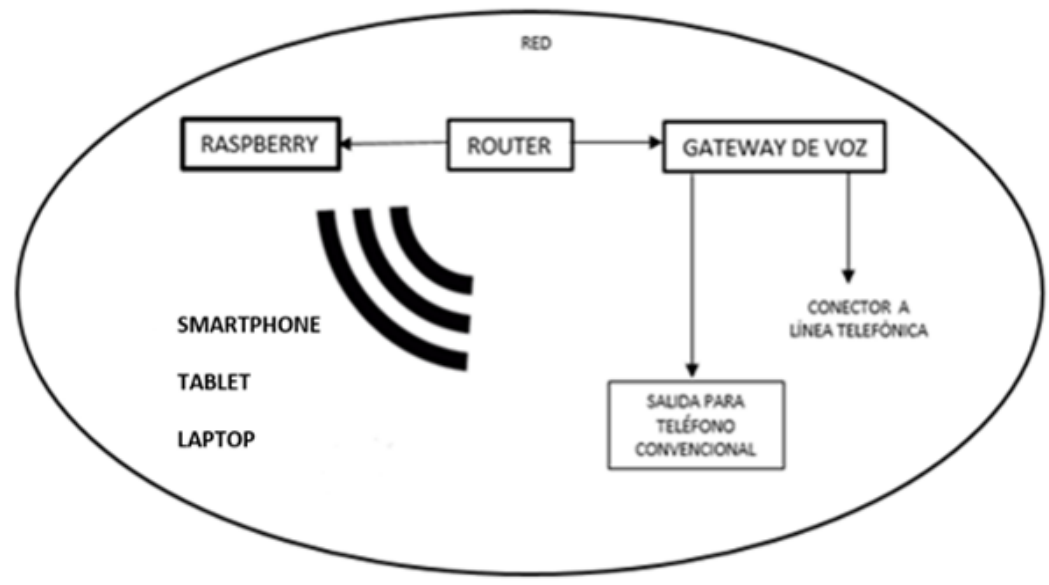

Fuente: Elaboración propia.

La Figura 1 muestra las conexiones necesaria de la tarjeta Raspberry Pi, el Gateway de voz y los diferentes dispositivos de comunicación de los huéspedes y personal del hotel. No será necesario que exista un teléfono en cada habitación ya que el sistema permite que cualquier teléfono móvil inteligente, computadora o Tablet se comuniquen entre si internamente , haciendo uso de la línea telefónica a través de diferentes aplicaciones gratuitas, también llamadas en general softphones, mediante tecnología VoIP.

Es importante tener en cuenta que dependiendo del tipo de Gateway se podrá adaptar cualquier teléfono analógico al sistema, en este caso se adaptó uno para La Recepción.

Figura 2. Esquema de conexión. Adaptado de (RASPBERRY PI 1 MODEL B, 2017)

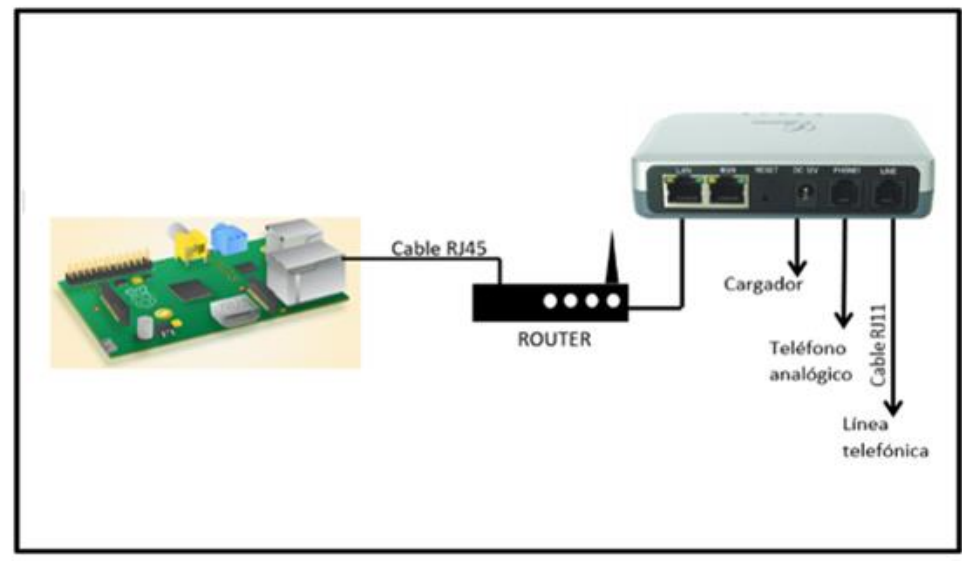

Fuente: (Grandstream, 2016). 
La Figura 2 indica la conexión del Gateway de voz Grandstream HT503 (Grandstream, 2016), el cual estará conectado a la línea telefónica para permitir llamadas desde y hacia la red pública, incluyendo al teléfono convencional en La Recepción, funcionando así como un usuario más.

Los diferentes clientes que lleguen al hotel e incluso el dueño del mismo y sus empleados podrán conectarse a una extensión con su respectiva clave. Para que exista un control y correcto uso del servicio telefónico, el sistema implementado incluye la opción de cambio de claves de una manera accesible exclusivamente para la administración del hotel.

Para el control de llamadas externas se adaptó y configuró en el sistema de la tarjeta Raspberry un servicio de control y facturación el cual registra los números marcados por cada usuario de la central, la duración y el costo por cada llamada, sea nacional o internacional.

\section{Configuración Raspberry}

La tarjeta Raspberry funciona específicamente con una tarjeta SD (Secure Digital) que cumple la función de disco duro (Hard Drive), por lo cual es necesario que disponga de la suficiente memoria, en este caso $8 \mathrm{~GB}$, ya que debe llevar montada una imagen iso escogida de la página de Raspberry con licencia pública. Se utilizó la imagen con licencia libre “raspbx-22-02-2015.iso" (Raspberry, 2017), la cual será grabada por medio de la aplicación de software libre Win32DiskImager, el sistema operativo que maneja es Debian e incluye el software de Asterisk.

Una vez que el sistema sea cargado en la tarjeta SD, se coloca la misma en la Raspberry P $i$, al encender el dispositivo se abrirá una interfaz para líneas de comandos, es necesario obtener del repositorio las diferentes actualizaciones de los paquetes disponibles en el sistema con acceso a internet. Una vez actualizado el sistema, se procede a la configuración de Asterisk, el cual ya viene instalado dentro de la imagen descargada anteriormente. 


\section{Configuración de llegada de mensajes de voz a mail}

Las llamadas entrantes a La Recepción del hotel que no tengan contestación, son las únicas que tendrán la opción de dejar mensajes de voz, los mismos que serán enviados al mail indicado en su configuración.

Debian por defecto tendrá instalado Exim4, que es un servicio para el envío de correos. Para su configuración se escribirá la siguiente línea en la pantalla de comandos:

>dpkg-reconfigure exim4-config

A continuación aparecerá una pantalla con una serie de preguntas, las cuales se responderán de acuerdo a las características que maneje el dominio del mail que se usará.

En el archivo con dirección /etc/exim4/passwd.client se configura los parámetros correctos del mail desde donde se enviaran los mensajes del correo de voz, en este caso la configuración utilizando una cuenta Gmail es la siguiente:

gmail-smtp.l.google.com:usuario@gmail.com:contraseña

*.google.com:usuario@gmail.com:contraseña

smtp.gmail.com:usuario@gmail.com:contraseña

Para la configuración del mail que recibirá los mensajes de voz se edita el archivo /etc/Asterisk/voicemail.conf donde se indica el número donde se marca para escuchar el mensaje de voz, la clave para poder acceder y el mail donde se recibe el mismo, agregando la siguiente línea:

Numero_para_mensaje_de_voz $\Rightarrow$ clave, Usuario, emailrecepción@gmail.com ; 


\section{Configuración de extensiones para central telefónica}

Asterisk posee sus archivos de configuración en la carpeta /etc/Asterisk los cuales van a ser modificados con el propósito de adaptarse a los requerimientos según el número de extensiones necesarias para el establecimiento. Los archivos que se modificaran son sip.conf y extensions.conf, se puede acceder a ellos mediante comandos en la consola.

En el archivo sip.conf se precisa el número de extensiones, se programarán los parámetros del canal para cada uno de los usuarios registrados en el archivo, como los puertos, codecs de sonido, audio, y diferentes tipos de conexión. Para la configuración de cada una de las extensiones asignadas se debe tomar en cuenta un contexto que se configura en el archivo extensions.conf, en este caso llamado "users" y utilizando los parámetros:

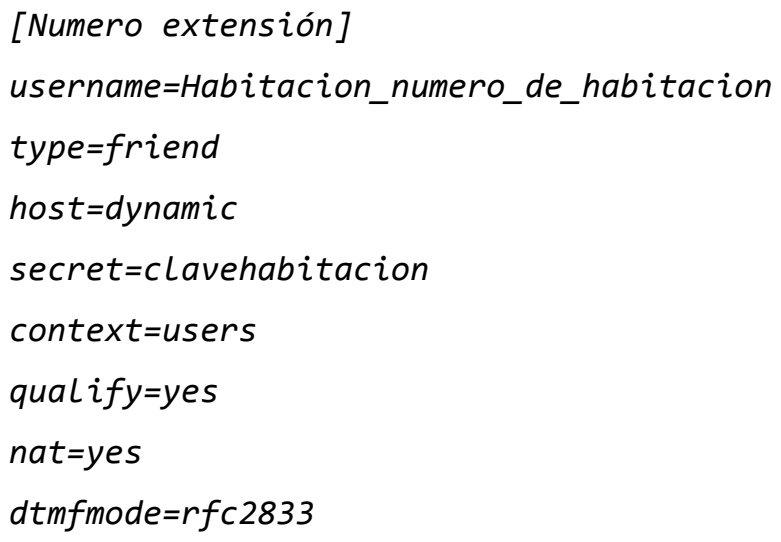

Para la extensión de La Recepción se toma en cuenta la configuración para almacenar mensajes de voz que se puedan dirigir de igual manera que al teléfono, al mail que se asigne, simplemente siguiendo el ejemplo anterior aumentando la línea mailbox= num@default al final de la configuración.

Es importante la configuración dos extensiones, una específicamente para permitir la salida de llamadas a la red telefónica y otra para recibir llamadas de la red telefónica.

En los archivos de extensions.conf se configura el plan de marcado, es donde se indicará el comportamiento de la central telefónica definiendo por medio de contextos, que acciones tomar cuando exista una llamada desde y hacia la red telefónica (PSTN) y cuando se realice 
una llamada entre extensiones internas. El contexto "users" es el que es llamado por cada extensión en el archivo sip.conf, en este caso se configurarán 25 extensiones bajo el mismo contexto, de las cuales 13 pertenecen a las habitaciones:

[users]

Exten $=>$ Num_de extensión, 1,Dial(SIP/ Num_de_extension, 20)

Para la extensión asignada a La Recepción en la configuración se le indica que la llamada que se dirige a La Recepción y si no recibe contestación, se redirigirá a una extensión asignada al propietario del hotel o empleado del mismo, si la llamada sigue sin recibir contestación por último se enviará a un buzón de voz:

exten $\Rightarrow>$ Num_extension_recepcion, 1,Dial(SIP/ Num_extension_recepcion, 20)

exten $\Rightarrow$ Num_extension_recepcion, 2,Goto(users, Num_extension_propietario,1)

exten =>Num_extension_recepcion,n,VoiceMail(num @default)

Cuando se realiza una llamada externa desde cualquier extensión se indica en la configuración que para la llamada se debe iniciar con un número específico para llamadas nacionales, otro para llamadas internacionales y otro para llamadas a celular seguido de cualquier cantidad de números entre 0 y 9 , dependiendo de las cifras de números requeridos. El sistema enviará el número sin el primer digito mediante la extensión sip al Gateway para que se conecte con la PSTN:

exten $\Rightarrow>7 x ., 1, \operatorname{Dial}(S I P / 9 / \$\{E X T E N: 1\})$

A continuación se configura el Interactive Voice Response (IVR) que funcionará al recibir llamadas de la red telefónica (PSTN), el IVR dará opciones para dirigir la llamada y comunicarse con las extensiones de las habitaciones o con La Recepción manejando el mismo contexto:

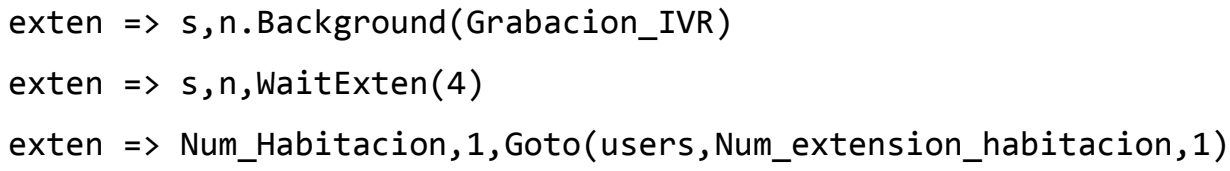




\section{Configuración de Gateway de voz HT503}

Para la implementación del sistema en el hotel se utilizó el Gateway GrandStream HT503

(Grandstream, 2016), el cual permitirá que se establezca comunicación hacia la PSTN con llamadas entrantes y salientes. Existen diferentes marcas y modelos de Gateway económicos que dan la posibilidad de adaptar teléfonos analógicos al sistema, dependiendo de cuantos se utilicen, en este caso solo se necesita de uno.

Figura 3. Puertos físicos de Gateway de voz

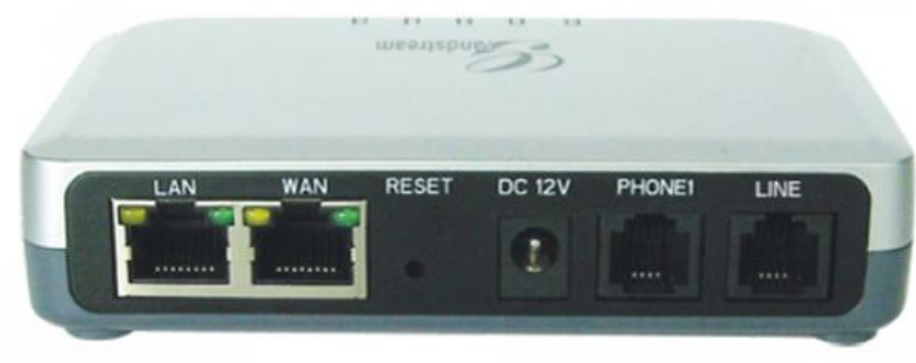

Fuente: (Grandstream, 2016).

El Gateway GrandStream HT503 cuenta con dos puertos analógicos, un FXS representado como PHONE y un FXO representado como LINE, en la Figura 3 están representados físicamente por las entradas PHONE y LINE, también cuenta con dos interfaces de tipo Ethernet.

Figura 4. Pestañas de configuración para Gateway de voz (Configuring the Grandstream HandyTone 503/HT-503, 2015).

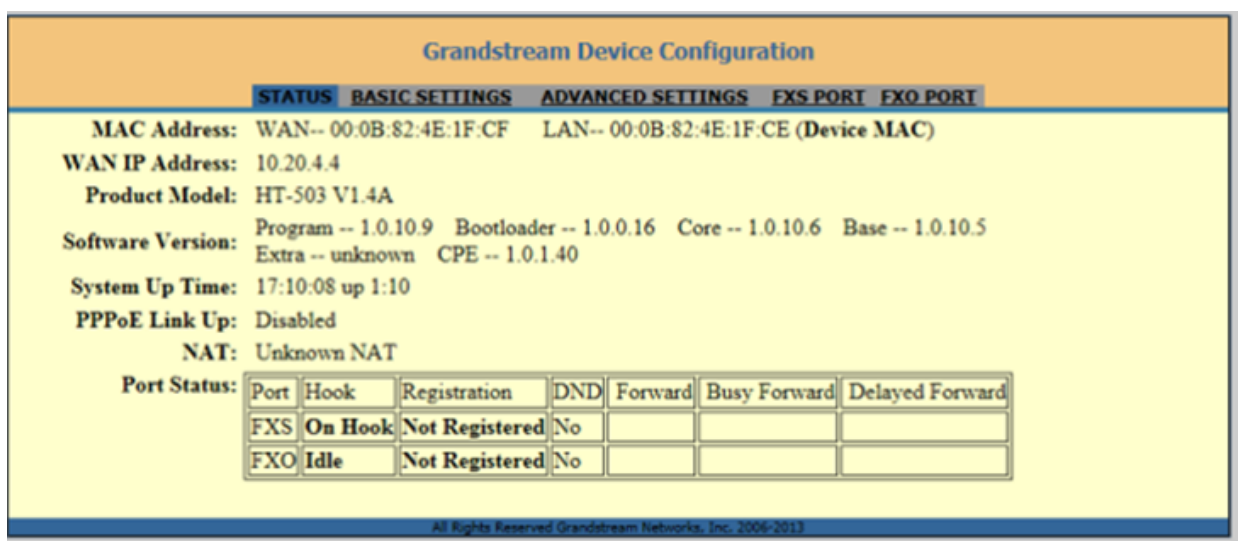


Para su configuración hay tres pestañas importantes como se muestra en la Figura 4, en Basic Settings se configuraran los parámetros de red del gateway, en FXS PORT se configura la extensión a donde se recibirán las llamadas, mientras que en la pestaña de FXO PORT se configura la extensión de donde saldrán las llamadas, tanto en los puertos FXS y FXO se ingresa la dirección IP (Internet Protocol) del servidor Asterisk.

\section{Servicio de control y tarifación para llamadas hacia la red convencional}

Dentro de la implementación de todo el sistema para la central telefónica es necesario el control de las llamadas externas de cada empleado y cliente.

Siguiendo los pasos de configuración de la página de Servitux (Servitux Servicios Informáticos SL., 2016), se configuró e instaló la aplicación de software libre OPEN Servitux Tarificador para Asterisk, no depende de la infraestructura de telefonía, lo único que necesita es el CDR (del inglés Call Detail Record) de Asterisk donde se almacena toda la información relativa a las llamadas.

La aplicación es muy sencilla de manejar ya que permite visualizar un registro que muestra las llamadas externas nacionales, internacionales y de celular de cada extensión registrada en el sistema, se puede controlar el número de extensiones, en el caso de que se crearan más o se eliminara alguna dentro de la central telefónica.

El software al ser configurable cuenta con varios beneficios, como registrar el precio de llamadas sea nacionales, internacionales o hacia números celulares, además el software calcula y muestra el costo total de cada llamada por extensión.

\section{Control de clave para extensiones}

Para cada extensión se tiene una clave asignada inicial, pero es necesario que estén en un contante cambio ya que el usuario de cada una de estas puede hacer un mal uso realizando llamadas que posiblemente no lleguen a ser cobradas. 
Para poder controlar el cambio de claves se programó en código php y html la búsqueda dentro del archivo sip.conf, que es donde se encuentran asignadas las claves, la línea "secret=clave_asignada" donde se reemplazará la clave asignada por cualquier clave diferente, se podrá ingresar a la interfaz gráfica desde cualquier computadora laptop o celular inteligente que se encuentre dentro de la red donde se pedirá que se ingrese la antigua clave y la nueva clave que se deseé asignar; el dueño o el encargado principal del hotel serán los únicos que tengan conocimiento de la dirección para acceder a la interfaz.

\section{Prueba de Carga}

Para la prueba de carga se pretende simular el envío de llamadas simultáneas con el software libre SIPp (SIPp, 2017).

SIPp se encarga de enviar un mensaje SIP-Invite al servidor Asterisk, mientras que Asterisk envía otro SIP-Invite al destino, Asterisk reenvía flujos de llamadas el cliente, SIPp al finalizar la transmisión envía un mensaje de Bye al servidor de Asterisk.

SIPp tienen un funcionamiento de tipo cliente/servidor, permite la creación de escenarios personalizados, definiendo flujos de llamadas, una vez implementada la central, se le debe instalar empezando por los siguientes comandos:

apt-get install c++ Libncurses5-dev Libpcap0.8-dev Libnet1-dev

wget http://surfnet.dl.sourceforge.net/sourceforge/sipp/sipp.3.1.src.tar.gz

Según se requiera se lo configurará tanto servidor y cliente, estos escenarios se los podrá encontrar en la página de SIPp (SIPp, 2017) donde hay archivos en formato xml según los escenarios que se requieran. Es importante incluir la siguiente línea en el archivo sip.conf para aceptar todas las llamadas sin el requerimiento de autentificación:

Allowguest=yes

Para la ejecución de las pruebas de carga se ejecutará el siguiente comando: 
./sipp -sf UAC.xml -s extension_cliente_asterisk - 1 num_llamadas_simultaneas -m numllamadas_enviar - $r$ llamadasxsegundo - trace_screen -trace_err -recv_timeout 400000 -t un $-n r$

Se utiliza trace_screen, para crear el archivo de registro con estadísticas, trace_shortmsg que se encarga de crear el archivo que envía y recibe mensajes SIP, trace_err se encarga del archivo de registro de errores, recv_timeout 400000 es el tiempo de espera en milisegundos.

Se realizó la prueba de estrés primero enviando 20 llamadas:

sipp -sf ./UAC.xmL -s 333 127.0.0.1:5080 - L 2 -m 20 -r 2 -trace_screen trace_shortmsg -trace_err-recv_timeout 400000 -t un - $n r$

Figura 5. Prueba SIPp con 20 llamadas.

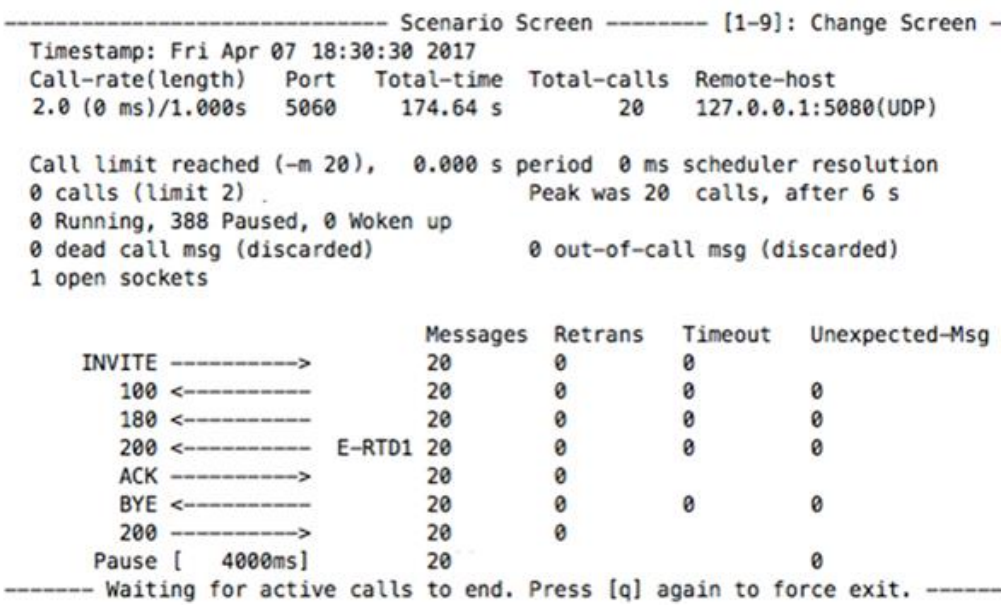

Fuente: Elaboración propia.

Se puede ver en la figura 5 que al sistema le tomó 174.64 segundos para procesar las 20 llamadas, no hubo ningún problema ya que completo el tráfico de llamadas enviadas, ahora se probará con 200 llamadas:

sipp -sf./UAC.xmL -s 333 127.0.0.1:5080 - L 200 -m 30 -r 200 -trace_screen trace_shortmsg -trace_err -recv_timeout 400000 -t un - nr 
Figura 6. Prueba SIPp con 200 llamadas.

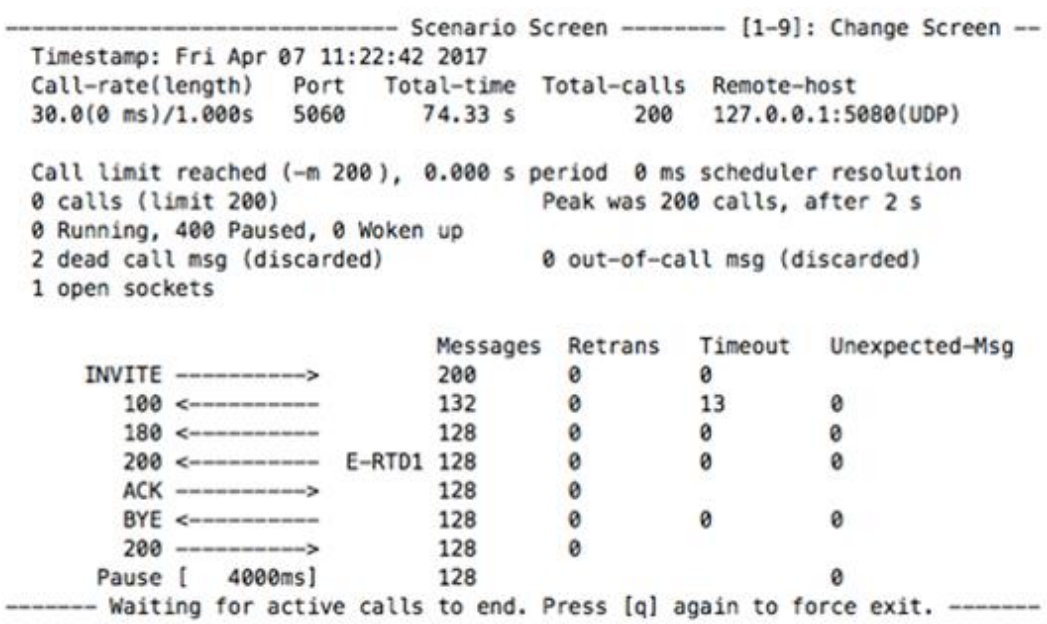

Fuente: Elaboración propia.

Se puede observar en la Figura 6 que no se completó el tráfico de 200 llamadas, se establecieron 128 simultáneamente, con lo que se concluye que 128 es la capacidad del sistema implementado según las características del mismo.

\section{Análisis de Costos Central Asterisk vs. Centrales de Marca}

Las centrales telefónicas como tal evitan la conexión de teléfonos de manera separada hacia la red telefónica pública, lo que conlleva al ahorro de cargos mensuales en la línea telefónica para quienes las emplean.

La central implementada basada en el software Asterisk da a lugar a un ahorro en comparación con otras centrales telefónicas privadas las cuales para sus costos dependen de su marca.

\section{Costo del Equipo}

Para su costo referencial se debe tomar en cuenta los equipos utilizados para el buen funcionamiento del sistema ya que se debe integrar software estable y hardware confiable, 
lo que garantizará el tráfico de llamadas, definiendo el costo total en la Tabla 2. De la central implementada se tienen las siguientes características:

Tabla 1. Costo de Central implementado.

\begin{tabular}{|c|c|c|}
\hline Hardware & Precio & Caracteristica \\
\hline Raspberry Pi modelo B & $\$ 65.00$ & $\begin{array}{c}\text { Puede manejar hasta 100 } \\
\text { extesiones }\end{array}$ \\
\hline $\begin{array}{c}\text { Gateway Grandstream } \\
\text { HT-503 }\end{array}$ & $\$ 80.00$ & 1 FXO, 1 FXS \\
\hline Cable RJ45 & $\$ 3.00$ & Incluido conector RJ45 \\
\hline Router & $\$ 60.00$ & TP-LINK \\
\hline TOTAL & $\$ 208$ & \\
\hline
\end{tabular}

Fuente: Elaboración propia.

Su licencia libre hace que no sea necesaria la actualización de la misma con cargo adicional y la información que se puede encontrar es muy amplia, la central funcionará correctamente sin desperdiciar recursos a un precio reducido en el caso de que el hardware sufra algún daño y sin ningún costo en caso de tratarse de daños o desconfiguración en software.

La central implementada ha pasado conectada sin que el equipo utilizado sufra de recalentamientos o el sistema en sí tienda a ponerse lento.

Se ha considerado las marcas de Avaya, CISCO y $3 \mathrm{COM}$ en la Tabla 2 como los principales competidores contra la central Asterisk, en sí, cualquier software libre es una amenaza para estas marcas. Las 3 marcas tienen centrales dirigidas a medianas y pequeñas empresas pero se manejan a un cierto costo dependiendo de las características de la central telefónica: 
Tabla 2. Costo de Centrales PANASONIC, AVAYA y 3CX .

\begin{tabular}{|l|l|l|}
\hline MARCA & PRECIO & CARACTERISTICA \\
\hline PANASONIC & $\$ 800-\$ 1189$ & $\begin{array}{l}\text { Central adaptable a } \\
\text { teléfonos analógicos y } \\
\text { dispositivos que cuenten } \\
\text { con softphone }\end{array}$ \\
\hline AVAYA & $\begin{array}{l}\$ 480 \text { (Precio depende de } \\
\text { País donde se lo requiera) }\end{array}$ & $\begin{array}{l}\text { Es necesario la compra de } \\
\text { licencia para cada teléfono } \\
\text { IP AVAYA. (AVAYA, } \\
\text { 2017) }\end{array}$ \\
\hline $3 \mathrm{CX}$ & $\$ 320$ & $\begin{array}{l}\text { Depende de las } \\
\text { características de la central } \\
\text { que se requiera ya que su } \\
\text { precio varía desde una } \\
\text { versión gratuita hasta una } \\
\text { versión Pro (3CX,2017) }\end{array}$ \\
\hline
\end{tabular}

Fuente: Elaboración propia.

Se debe tomar en cuenta que no manejan licencia libre, por lo que es necesaria su renovación. Dependiendo del proveedor se paga por cada extensión adicional que se requiera.

\section{Resultados en Análisis MOS}

Después de la implementación del sistema es necesario un análisis de la calidad del servicio, entender como los clientes perciben la calidad al realizar sus llamadas, esto se lo hará utilizando el análisis MOS (Mean Opinion Score), para situaciones de conversación MOSc (Mean Opinion Score conversational), la comunicación será evaluada sobre una escala de calidad ACR (Absolute Category Rating) definida según la UIT-T P.800 (Itu.int., 2016) Excelente $=5$, Buena $=4$, Regular $=3$, Mediocre $=2$, Mala $=1$.

Se evaluará la calidad de comunicación interna entre extensiones y externa con la salida de llamadas a la PSTN en la situación más realista. 
Para la comunicación interna, el análisis por ser conversacional será de 2 participantes instalados en diferentes habitaciones manteniendo una conversación estructurada, es decir, que tenga un inicio, un desarrollo y un final. Por observación las conversaciones en el hotel están compuestas por un saludo, un pedido por parte del huésped, una respuesta del empleado del hotel y para finalizar una despedida. Los participantes al finalizar la conversación califican la comunicación según la escala antes mencionada y se evalúan la comunicación con respecto a la degradación de la voz que pueda existir debido al eco, ruido o cualquier interrupción que pueda presentarse.

En 7 días, de las 13 habitaciones se tomó una muestra de 38 personas que independientemente del tiempo que se quedaron en el hotel utilizaron el sistema de comunicación interna, la calificación de calidad tuvo los siguientes resultados:

Figura 7. Representación MOS comunicación interna.

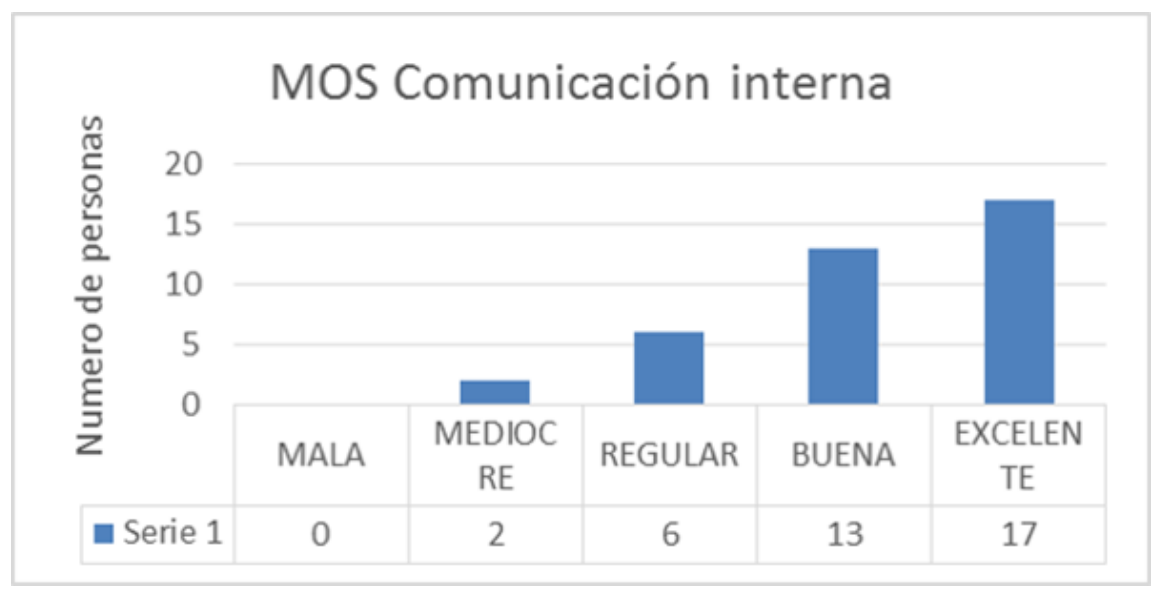

Fuente: Elaboración propia.

Según la Figura 7 existe la posibilidad de que la comunicación no se haya realizado con éxito en todas las llamadas, la central IP siempre estará expuesta a ciertas interferencias, interrupciones o distorsiones, comparando se puede observar que la tendencia en la opinión de la gente está entre BUENA y EXCELENTE. 
Para la comunicación externa se buscó evaluar si los usuarios pudieron utilizar el sistema para realizar llamadas desde su celular o tablet con la aplicación softphone hacia cualquier número de teléfono, ya sea nacional, internacional o celular y así poder tener una conversación con su respectivo inicio, desarrollo y fin.

Figura 8. Representación MOS, comunicación externa hacia la red telefónica.

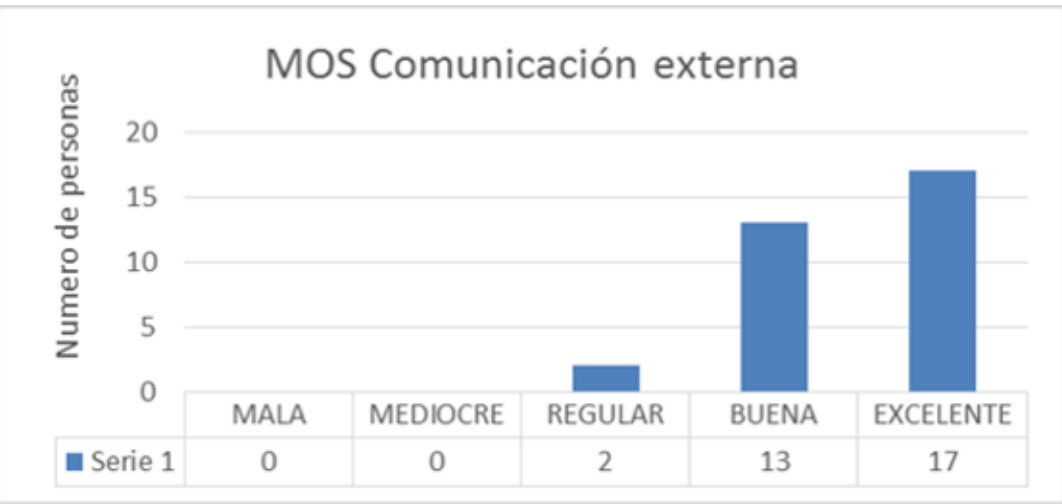

Fuente: Elaboración propia.

Entre las distintas opiniones de las personas que participaron en las pruebas de calidad la mayoría manifestó que, encontraron un poco de dificultad con el manejo del softphone pero finalmente lograron establecer comunicación con éxito, las personas que no lograron establecer comunicación calificaron la comunicación como regular ya que no lograron configurar su celular.

El sistema en su mayor parte tiende a ser BUENO o EXCELENTE, es así como se puede describir al sistema exitoso y es importante tener en cuenta la adaptabilidad del cliente dependiendo del negocio donde se busque implementar la central telefónica. 


\section{Discusión y Conclusiones}

Dentro de un negocio es importante mantener contacto directo con los clientes de manera constante, por lo que adquirir un sistema de comunicación se ha vuelto imprescindible. La tecnología IP ofrece varias ventajas, ya que optimiza recursos a través de la misma red de Internet, para la transmisión de voz no requiere la dedicación o pago exclusivo de una red específica como generalmente se hacía con la telefonía tradicional, todo esto permite que haya una reducción de infraestructura, alojando todos estos servicios en una "Centralita" virtual a la cual se le puede añadir nuevas y mejores funcionalidades que con la telefonía tradicional eran inalcanzables o se presentaban costos bastante elevados para lograrlo.

Con la implementación de la Central Telefónica basada en Asterisk no se corre riesgo de que llegue a ser obsoleta por alguna falta de funcionalidad o necesidad ya que son adaptables a cualquier sistema, incluso a la telefonía antigua, permitiendo que la instalación de la misma no sea un problema sino más bien una solución que a largo plazo puede ser actualizada en cualquier momento.

En las diferentes fases del proyecto, se planificaron las posibles soluciones tanto en software y hardware para desarrollar un sistema de comunicación completo, sin embargo su configuración por ser programación de código abierto de licencia libre es susceptible a fallos, en sí vulnerable a errores humanos.

El sistema implementado no solo ofrece comunicación entre los diferentes terminales de red, también ofrece servicios de valor añadido como seguridad para cada extensión con manejo de claves y control de llamadas tanto internas como externas, dependiendo del negocio ofrece también sistema de facturación, con todo esto se demuestra que para las empresas que no tienen muchos recursos pueden acceder sin ningún problema a sistemas de alta calidad utilizando sistemas VoIP que terminan siendo igual o mejor que aquellos que utilizan una red telefónica tradicional totalmente analógica. 
El diseño de la "Centralita" se ha basado en el uso de software libre y gratuito con una notable disminución de gastos en comparación con otras Centrales IP de marca reconocida; es una gran competencia por los diferentes servicios que se pueden incorporar sin ningún costo, concluyendo que se puede introducir en el mercado un recurso con las mismas o mejores características que las centralitas usuales de los grandes proveedores a un precio más económico.

$\mathrm{Al}$ implementar el sistema con software de código abierto se puede controlar la utilización de los diferentes servicios de telefonía IP, de tal manera que cada cliente sea consiente y haga un uso racional del servicio.

\section{Bibliografía}

Asterisk.org. (2017). Homepage. [online] Available at: http://www.asterisk.org/ [Accessed 12 Oct. 2016].

AVAYA. (2017). [online] Available at: https://www.avaya.com/en/products/ [Accessed 3 April. 2017].

Configuring the Grandstream HandyTone 503 (HT-503) [Imagen pag 2]. (2015). Available at:

http://files.inductiveautomation.com.s3.amazonaws.com/whitepapers/GrandstreamHT503SetupGuide\%20v1.pdf [Accessed 12 Oct. 2016].

Dong, Q. (2011). Research on the Performance of Asterisk-Based Media Gateway. Fourth International Symposium on Knowledge Acquisition and Modeling, 2011.

Estrada, J. A., Peláez, D., \& Tipantuña, J. C., (2015). Performance Analysis of a Raspberry Pi Based IP Telephony Platform. Revista Politécnica, vol 36, 2016 , $72-77$.

Grandstream, (2016). [online] Available at: 
http://www.grandstream.com/products/gateways-and-atas/analog-telephoneadaptors/product/handytone-503 [Accessed 12 Oct. 2016].

Gupta, P., Agrawal, N., \& Qadeer, M. (2013). GSM and PSTN gateway for asterisk EPBX. Tenth International Conference on Wireless and Optical Communications Networks (WOCN), 2013.

Itu.int. (2016). Métodos de determinación subjetiva de la calidad de transmisión. [online] Available at: https://www.itu.int/rec/T-REC-P.800-199608-I/es [Accessed 12 Jan. 2017].

Li, C., Li, H., Wang, K. \& Nan, K. (2011). Research and Implementation of Unified Communications System based on Elastix. 7th International Conference on Wireless Communications, Networking and

Mobile Computing, 2011.

Masudur, M., \& Sarwar, N. (2014). VoIP Implementation Using Asterisk PBX. IOSR Journal of Business and Management (IOSR-JBM), vol 15, 2014, 47-53.

Murkute, P., \& Deshmukh, V. (2015). Implementing the VOIP Communication Principles using Raspberry Pi as Server. International Journal of Computer Applications, vol $124,2015,34-38$.

Pelaéz, D., \& Tipantuña, C. (2014). Servidor de comunicaciones unificadas con Raspberry Pi y Micro-Elastix. MASKANA, I+D+ingeniería, 2014, 293-301.

Qadeer,M.,\& Imran, A. (2008). Asterisk Voice Exchange: An Alternative to Conventional EPBX. International Conference on Computer and Electrical Engineering,2008, 652656. 
Raspberry, (2017). [online] Available at: http://www.raspberry-asterisk.org/downloads/ [Accessed 12 Oct. 2016].

RASPBERRY PI 1 MODEL B [Imagen] (2017). Available at: https://www.raspberrypi.org/products/model-b/ [Accessed 21 Oct. 2016].

Servitux Servicios Informáticos SL. (2016). Tarificador Asterisk - Servitux Servicios Informáticos SL. [online] Available at: https://www.servitux.es/informacion/tarificador-asterisk/ [Accessed 14 Oct. 2016].

Sinaeepourfard, A., \& Mohamed, H. (2011). Comparison of VoIP and PSTN Services by Statistical Analysis. IEEE Student Conference on Research and Development,2012, 459-461.

SIPp. (2017). [online] Available at: http://sipp.sourceforge.net/doc/reference.html\#Installation [Accessed 3 April. 2017].

Villacis, D., Acosta, F., \& Lara, R. (2013). Performance Analysis of VoIP Services over WiFi-based systems. IEEE Colombian Conference on Communications and Computing (COLCOM), 2013, 1-6.

3CX. (2017). [online] Available at: https://www.3cx.es/centralita-telefonica/comparacioncaracteristicas/ [Accessed 3 April. 2017]. 